\title{
A NOTE ON ALMOST SUBNORMAL SUBGROUPS OF LINEAR GROUPS
}

\author{
B. A. F. WEHRFRITZ
}

(Communicated by Ronald M. Solomon)

\begin{abstract}
Following Hartley we say that a subgroup $H$ of a group $G$ is almost subnormal in $G$ if there is a series of subgroups $H=H_{0} \leq H_{1} \leq \cdots \leq H_{r}=G$ of $G$ of finite length such that for each $i<r$ either $H_{i}$ is normal in $H_{i+1}$ or $H_{i}$ has finite index in $H_{i+1}$. We extend a result of Hartley's on arithmetic groups (see Theorem 2 of Hartley's Free groups in normal subgroups of unit groups and arithmetic groups, Contemp. Math., vol. 93, Amer. Math. Soc., Providence, RI, 1989, pp. 173-177) to arbitrary linear groups. Specifically, we prove: let $G$ be any linear group with connected component of the identity $G^{0}$ and unipotent radical $U$. If $H$ is any soluble-by-finite, almost subnormal subgroup of $G$ then $\left[H \cap G^{0}, G^{0}\right] \leq U$.
\end{abstract}

Following Hartley [1] we say that a subgroup $H$ of a group $G$ is almost subnormal in $G$, and write $H$ asn $G$ for short, if there is a series of subgroups

$$
H=H_{0} \leq H_{1} \leq \cdots \leq H_{r}=G
$$

of $G$ of finite length such that for each $i<r$ either $H_{i}$ is normal in $H_{i+1}$ or $H_{i}$ has finite index in $H_{i+1}$. Theorem 2 of [1] reads as follows. Let $H$ be a connected reductive algebraic $\mathbb{Q}$-group and $\Gamma$ an arithmetic subgroup of $H$. Then $\Gamma$ contains a normal subgroup $\Gamma_{0}$ of finite index such that if $\Delta$ is an almost subnormal subgroup of $\Gamma$, then either $\Delta$ contains a nonabelian free group or $\left[\Delta \cap \Gamma_{0}, \Gamma\right]=\langle 1\rangle$.

In this note we show that this result can be viewed as a special case of a basically elementary result about arbitrary linear groups. Note first that by Tits's theorem $[2,10.17]$ either the $\Delta$ above contains a nonabelian free subgroup or $\Delta$ is soluble-by-finite. Thus we are concerned with soluble-by-finite almost subnormal subgroups of linear groups.

Throughout this note $F$ denotes a field, $n$ a positive integer, and $G$ a subgroup of $\mathrm{GL}(n, F)$. Then $G$ carries its Zariski topology and topological terms below refer to this topology. $G$ has a unique minimal closed subgroup of finite index; we denote this subgroup by $G^{0}$. Also $u(G)$ denotes the unipotent radical of $G$. Our main result is the following.

Proposition 1. Let $G$ be a subgroup of $\mathrm{GL}(n, F)$ and $H$ a soluble-by-finite, almost subnormal subgroup of $G$. Then $\left[H \cap G^{0}, G^{0}\right] \leq u\left(G^{0}\right)$.

Received by the editors May 6, 1991.

1991 Mathematics Subject Classification. Primary 20H20, 20 E15. 
If $\Gamma$ is as in [1, Theorem 2] quoted above, then the centre of $\Gamma^{0}$ lies in the centre of $\Gamma$ (indeed of $H$ ) and $u\left(\Gamma^{0}\right)=u(\Gamma)=\langle 1\rangle$. Consequently Hartley's theorem follows from Tits's theorem and Proposition 1 (with $\Gamma^{0}$ for $\Gamma_{0}$ ). (These facts about $\Gamma$ are easy to deduce from the first part of Hartley's proof of [1, Theorem 2].) If we recast Proposition 1 in the format of [1] we obtain

Corollary. Let $H$ asn $G \leq \operatorname{GL}(n, F)$. Suppose either that $\operatorname{char} F=0$ or that the entries of the elements of $G$ lie in some finitely generated subring of $F$. Then either $H$ contains a noncyclic free subgroup or $\left[H \cap G^{0}, G^{0}\right] \leq u\left(G^{0}\right)$.

In characteristic zero we can strengthen the conclusion of Proposition 1.

Proposition 2. There exists an integer-valued function $f(n)$ of $n$ only such that whenever $G \leq \mathrm{GL}(n, F)$ with $\mathrm{char} F=0$ there is a closed normal subgroup $C$ of $G$ with $(G: C) \leq f(n)$ such that if $H$ asn $G$ then either $H$ contains a noncyclic free subgroup or $[H \cap C, C] \leq u(C)$.

Although we have been discussing almost subnormal subgroups, the core of our proofs is that one needs only consider characteristic subgroups. This is the content of the following proposition.

Proposition 3. Let $G$ be a subgroup of $\mathrm{GL}(n, F)$. Then $G$ has a unique maximal soluble-by-finite, almost subnormal subgroup $T$ and $T$ contains every soluble-by-finite, almost subnormal subgroup of $G$.

\section{THE PROOFS}

1. Let $G \leq \mathrm{GL}(n, F)$. Then $G$ has a unique maximal soluble-by-finite normal subgroup $T$. The subgroup $T$ contains every soluble-by-finite normal subgroup of $G$.

Proof. The product $S$ of the soluble normal subgroups of $G$ is soluble and closed in $G$, see [2, 3.8 and 5.11]. Let $T$ be the product of the soluble-by-finite normal subgroups of $G$. Then $T / S$ is an $F C$-group and hence is centre-byfinite, see [2, 5.5 and 5.14]. Consequently, $T$ is soluble-by-finite, $T / S$ is finite, and the lemma is proved.

2. Let $H$ asn $G \leq \operatorname{GL}(n, F)$. Then $u\left(H^{0}\right) \leq u\left(G^{0}\right) \leq u(G)$ and $u(H)^{0} \leq$ $u(G)^{0}$. If $\operatorname{char} F=0$ then $u(H) \leq u(G)$.

Proof. Let $H=H_{0} \leq H_{1} \leq \cdots \leq H_{r}=G$ be as in the definition above of the statement ' $H$ asn $G$.' If $H$ is normal in $H_{1}$ then $H^{0}$ is normal in $H_{1}^{0}$; hence $u\left(H^{0}\right)$ is normal in $H_{1}^{0}$ and so $u\left(H^{0}\right) \leq u\left(H_{1}^{0}\right)$. Suppose $\left(H_{1}: H\right)$ is finite. Use a star to denote closures of subsets in $H_{1}$. Then $H^{0 *}$ is connected and $\left(H_{1}: H^{0 *}\right) \leq\left(H_{1}: H^{0}\right)$ is also finite. Consequently, $H^{0 *}=H_{1}^{0}$. Also $u\left(H^{0}\right)^{*}$ is unipotent and normal in $H^{0 *}$, see [2, 5.9 and 1.21]. Therefore,

$$
u\left(H^{0}\right) \leq u\left(H^{0}\right)^{*} \leq u\left(H^{0 *}\right)=u\left(H_{1}^{0}\right) .
$$

We have now proved that in all cases $u\left(H^{0}\right) \leq u\left(H_{1}^{0}\right)$. The same argument yields that $u\left(H_{i}^{0}\right) \leq u\left(H_{i+1}^{0}\right)$ for each $i$ and hence $u\left(H^{0}\right) \leq u\left(G^{0}\right)$.

Now $u(H)^{0} \leq H^{0}$, so $u(H)^{0} \leq u\left(H^{0}\right) \leq u\left(G^{0}\right) \leq u(G)$. It is also connected, so $u(H)^{0} \leq u(G)^{0}$. If $\operatorname{char} F=0$ then unipotent subgroups of $\operatorname{GL}(n, F)$ are connected and $u(H)=u(H)^{0} \leq u(G)^{0}=u(G)$. 
3. Suppose $H$ asn $G \leq \mathrm{GL}(n, F)$ with $H$ soluble-by-finite. Then $H^{G}=$ $\left\langle g^{-1} h g: h \in H, g \in G\right\rangle$ is also soluble-by-finite.

Proof. We may assume that $F$ is algebraically closed. Let $H=H_{0} \leq H_{1} \leq$ $\cdots \leq H_{r}=G$ be as in the definition of $H$ asn $G$. We induct on $r$. If $r=0$ there is nothing to prove, so assume otherwise and set $K=H_{r-1}$. By induction $H^{K}$ is soluble-by-finite. Replacing $H^{K}$ by $H$ we may assume that $H$ is normal in $K$. If $K$ is normal in $G$ then $H^{G}$ is soluble-by-finite by [5, 5.1]. Hence consider the case $(G: K)<\infty$. Replace $H$ and $K$ by their closures in $G$, so now $H$ and $K$ are closed subgroups of $G$.

Certainly $u(G)$ is soluble and $G / u(G)$ embeds into $\operatorname{GL}(n, F)$ in the standard way. Thus we may pass to $G / u(G)$ and assume that $u(G)=\langle 1\rangle$. In particular by 2 we now have $u\left(H^{0}\right)=\langle 1\rangle$. It follows from the Lie-Kolchin theorem [2,5.8] that $H^{0}$ is abelian. Now $K / u(K)$ has a faithful completely reducible representation in $\operatorname{GL}(n, F)$, see [2, Chapter 1], and hence [2, 1.12 and 5.4] the centralizer $C_{K}\left(H^{0}\right)$ is closed in $K$ of finite index. So too is $C_{K}\left(H / H^{0}\right)$, see $[2,5.10]$. Hence $G^{0}=K^{0}$ stabilizes the series $H \supseteq H^{0} \supseteq\langle 1\rangle$ and consequently $L=\left(G^{0}\right)^{\prime}$ centralizes $H$. Thus $H$ lies in the normal subgroup $C_{G}(L)$ of $G$ and hence $H^{G} \leq C_{G}(L)$. Finally $G^{0} \cap C_{G}(L)$ is soluble and of finite index in $C_{G}(L)$. The proof is complete.

4. The Proof of Proposition 3. Let $T$ be as in 1. The result then follows from 1 and 3.

5. Suppose $H$ asn $G \leq \mathrm{GL}(n, F)$ with $H$ soluble-by-finite and $u(G)=\langle 1\rangle$. Then $\left[H \cap G^{0}, G^{0}\right]=\langle 1\rangle$.

Proof. Let $T$ be as in Proposition 3. Then it suffices to prove that $\left[T \cap G^{0}, G^{0}\right]=\langle 1\rangle$. We may assume that $F$ is algebraically closed and, since $u(G)=\langle 1\rangle$, that $G$ is completely reducible. Then $T^{0}$ is abelian and $\left(G: C_{G}\left(T^{0}\right)\right)$ divides $n$ ! by [2,5.11, 5.8, and 1.12]. Certainly $T$ and $T^{0}$ are closed in $G$. Hence $L=C_{G}\left(T^{0}\right) \cap C_{G}\left(T / T^{0}\right)$ is a closed normal subgroup of $G$ of finite index. By stability theory, $L / C_{L}(T \cap L)$ embeds into

$$
\operatorname{Der}\left((T \cap L) /\left(T^{0} \cap L\right), T^{0} \cap L\right)=\operatorname{Hom}\left((T \cap L) /\left(T^{0} \cap L\right), T^{0} \cap L\right),
$$

since $T^{0} \cap L$ is central in $T \cap L$. Also $(T \cap L) /\left(T^{0} \cap L\right)$ is finite and $T^{0}$ is abelian with its torsion subgroup of finite rank (at most $n$, see [2, 2.2]). Hence this Hom group is finite and therefore $C=C_{L}(T \cap L)$ is a closed normal subgroup of $G$ of finite index. Consequently we have $G^{0} \leq C$ and $[T \cap C, C] \leq[T \cap L, C]=\langle 1\rangle$. The proof is complete.

6. The Proof of Proposition 1. There is a continuous homomorphism $\phi$ of $G$ into $\mathrm{GL}(n, F)$ with kernel $u(G)$ and image completely reducible. Then $(G \phi)^{0} \supseteq G^{0} \phi$. Also $\left[H \phi \cap(G \phi)^{0},(G \phi)^{0}\right]=\langle 1\rangle$ by 5 . Therefore $\left[H \cap G^{0}, G^{0}\right] \leq$ $\operatorname{ker} \phi \cap G^{0}=u(G) \cap G^{0}=u\left(G^{0}\right)$.

7. The Proof of Proposition 2. We modify the proof of 5. Again we may assume that $F$ is algebraically closed and that $G$ is completely reducible. Then $T$ has a closed diagonalizable subgroup $A$ normal in $G$ such that $(T: A)$ is bounded by an integer-valued function of $n$ only $[2,10.11 ; 3$, Proposition 1]. The proof of 5 yields that if $L=C_{G}(A) \cap C_{G}(T / A)$ and $C=C_{L}(T \cap L)$, then $C$ is 
a closed normal subgroup of $G$ with $(G: C)$ bounded by an integer-valued function of $n$ only and $[T \cap C, C]=\langle 1\rangle$.

8. The corollary to Proposition 1 is an immediate consequence of Proposition

1, Tits's theorem, and the following, no doubt well-known, fact:

If $H$ is a soluble-by-periodic subgroup of $\operatorname{GL}(n, R)$, where $R$ is a finitely generated integral domain, then $H$ is soluble-by-finite.

To see this note first that $H$ is soluble by locally-finite by $[2,5.9,5.11,6.4$, and 4.9]. Suppose first that $H$ is absolutely irreducible. Then by [2, 1.12] the group $H$ has a centre by locally-finite normal subgroup $K$ of finite index. Then $K^{\prime}$ is locally finite. Since $u\left(K^{\prime}\right) \leq u(H)=\langle 1\rangle$, it follows from [2, 4.8] that $K^{\prime}$ is finite. Consequently $H$ is soluble-by-finite. In general, by adjoining the (finitely many) entries and the inverse determinant of a suitable change-ofbasis matrix to $R$, we may assume that $H / u(H)$ is isomorphic to an absolutely completely reducible subgroup of $\mathrm{GL}(n, R)$. Then $H / u(H)$ is soluble-by-finite by the first case and consequently so is $H$.

9. Remarks. (i) Consider the situation of 5. Clearly we cannot prove in general that $\left[H, G^{0}\right]=\langle 1\rangle$, for we can choose $H=G$ and $G$ not centre-by-finite. However, we cannot even show that $G^{0}$ normalizes $H$, i.e., that $\left[H, G^{0}\right] \leq H$. For the infinite dihedral group,

$$
G=\left\langle x, y \mid x^{y}=x^{-1}, y^{2}=1\right\rangle
$$

has an embedding in $\operatorname{GL}(2, \mathbb{Q})$ (and also in $\operatorname{GL}(4, \mathbb{Z})$ ) with $G^{0}=\langle x\rangle$ and $u(G)=\langle 1\rangle$. Set $H=\left\langle x^{3}, y\right\rangle$. Then $(G: H)=3$ and so $H$ asn $G$. Also

$$
H\left[H, G^{0}\right] \geq\left\langle x^{3}, y,[y, x]=x^{2}\right\rangle=G .
$$

(ii) There is no analogue of Proposition 2 if $\operatorname{char} F=p>0$. For if $G$ is a finite simple linear group of degree $n$ and characteristic $p$, then the order of $G$ is not boundable and, since $G$ is an allowable choice for $H$, the only possibility for $C$ is $\langle 1\rangle$.

(iii) If char $F=0$ in 3 then $H^{G}$ has a soluble normal subgroup with finite index bounded by a function of $n$ only $[2,10.11]$. If char $F>0$ this conclusion is false. One can at least prove the following (cf. $[5,5.1])$.

Let $H$ asn $G \leq \mathrm{GL}(n, F)$. Suppose $H$ has a soluble normal subgroup of finite index $m$. Then $H^{G}$ has a soluble normal subgroup with index bounded by a function of $m, n$ and the indices $\left(H_{i+1}: H_{i}\right)$ for those $i$ for which $H_{i}$ is not normal in $H_{i+1}$. (The $H_{i}$ here are as in the definition of $H$ asn $G$.)

(iv) Using the techniques and main theorem of $[4, \S 6]$, including a converse of $[4,6.4]$, one can extend the results of this note as follows.

Let $R$ be a commutative ring, $M$ a Noetherian $R$-module, and $G$ a group of $R$-automorphisms of $M$.

(a) There exists a normal subgroup $C$ of $G$ of finite index such that whenever $H$ is a soluble-by-finite, almost subnormal subgroup of $G$, we have $[H \cap C, C] \leq u(C)$.

(b) If $R$ is finitely generated as a ring and $C$ is as in (a), then for every almost subnormal subgroup $H$ of $G$ either $H$ contains a noncyclic free subgroup or $[H \cap C, C] \leq u(C)$.

(c) $G$ has a unique maximal soluble-by-finite, almost subnormal subgroup $T$ and $T$ contains every soluble-by-finite, almost subnormal subgroup of $G$. 


\section{REFERENCES}

1. B. Hartley, Free groups in normal subgroups of unit groups and arithmetic groups, Contemp. Math., vol. 93, Amer. Math. Soc., Providence, RI, 1989, pp. 173-177.

2. B. A. F. Wehrfritz, Infinite linear groups, Springer-Verlag, Berlin, 1973.

3. __, On the Lie-Kolchin-Mal'cev theorem, J. Austral. Math. Soc. Ser. A 26 (1978), 270276.

4. __ Lectures around complete local rings, Queen Mary College Math. Notes, London, 1979.

5. __ Wielandt's subnormality criterion and linear groups, J. Algebra 67 (1980), 491-503.

School of Mathematical Sciences, Queen Mary and Westfield College, Mile End RoAD, LoNDON E1 4NS, Great BRITAIN 